OPEN ACCESS

Edited by:

Woon-Man Kung,

Chinese Culture University, Taiwan

Reviewed by:

Lee Wei Lim,

The University of Hong Kong,

Hong Kong

Hirokazu Kawagishi,

Shizuoka University, Japan

${ }^{*}$ Correspondence:

Chin-Chu Chen

gkbioeng@grapeking.com.tw

David Pei-Cheng Lin

pcl@csmu.edu.tw

Received: 26 February 2020 Accepted: 07 May 2020

Published: 03 June 2020

Citation:

Li I-C, Chang H-H, Lin C-H,

Chen W-P, LU T-H, Lee L-Y,

Chen $Y-W$, Chen $Y-P$, Chen $C-C$ and Lin DP-C (2020) Prevention of Early

Alzheimer's Disease by Erinacine A-Enriched Hericium erinaceus Mycelia Pilot Double-Blind

Placebo-Controlled Study.

Front. Aging Neurosci. 12:155.

doi: 10.3389/fnagi.2020.00155

\section{Prevention of Early Alzheimer's Disease by Erinacine A-Enriched Hericium erinaceus Mycelia Pilot Double-Blind Placebo-Controlled Study}

\author{
I-Chen Li', Han-Hsin Chang', Chuan-Han Lin ${ }^{3}$, Wan-Ping Chen ${ }^{1}$, Tsung-Han Lu', \\ Li-Ya Lee', Yu-Wen Chen', Yen-Po Chen', Chin-Chu Chen ${ }^{1,4,5,6 *}$ and \\ David Pei-Cheng Lin ${ }^{3,7 *}$

\begin{abstract}
'Biotech Research Institute, Grape King Bio Ltd., Taoyuan City, Taiwan, ${ }^{2}$ Department of Nutrition, Chung Shan Medical University, Taichung City, Taiwan, ${ }^{3}$ Department of Medical Laboratory and Biotechnology, Chung Shan Medical University, Taichung City, Taiwan, ${ }^{4}$ Institute of Food Science and Technology, National Taiwan University, Taipei City, Taiwan, ${ }^{6}$ Department of Bioscience Technology, Chung Yuan Christian University, Taoyuan City, Taiwan, ${ }^{7}$ Department of Ophthalmology, Chung Shan Medical University Hospital, Taichung City, Taiwan
\end{abstract} \\ ${ }^{5}$ Department of Food Science, Nutrition and Nutraceutical Biotechnology, Shih Chien University, Taipei City, Taiwan,
}

Objective: To investigate the efficacy and safety of three $H$. erinaceus mycelia (EAHE) capsules (350 mg/capsule; containing $5 \mathrm{mg} / \mathrm{g}$ erinacine A active ingredient) per day for the treatment of patients with mild Alzheimer's Disease (AD).

Methods: This study comprised a 3-week no-drug screening period, followed by a 49-week double-blind treatment period with 2-parallel groups in which eligible patients were randomized to either three $5 \mathrm{mg} / \mathrm{g}$ EAHE mycelia capsules per day or identical appearing placebo capsules. Cognitive assessments, ophthalmic examinations, biomarker collection, and neuroimaging were followed throughout the study period.

Results: After 49 weeks of EAHE intervention, a significant decrease in Cognitive Abilities Screening Instrument score was noted in the placebo group, a significant improvement in Mini-Mental State Examination score was observed in the EAHE group and a significant Instrumental Activities of Daily Living score difference were found between the two groups. In addition, EAHE group achieved a significantly better contrast sensitivity when compared to the placebo group. Moreover, only the placebo group observed significantly lowered biomarkers such as calcium, albumin, apolipoprotein E4, hemoglobin, and brain-derived neurotrophic factor and significantly elevated alpha1antichymotrypsin and amyloid-beta peptide 1-40 over the study period. Using diffusion tensor imaging, the mean apparent diffusion coefficient (ADC) values from the arcuate fasciculus region in the dominant hemisphere significantly increased in the placebo group while no significant difference was found in the EAHE group in comparison to their baselines. Moreover, ADC values from the parahippocampal cingulum region in the dominant hemisphere significantly decreased in the EAHE group whereas no significant 
difference was found in the placebo group when compared to their baselines. Lastly, except for four subjects who dropped out of the study due to abdominal discomfort, nausea, and skin rash, no other adverse events were reported.

Conclusion: Three 350 mg/g EAHE capsules intervention for 49 weeks demonstrated higher CASI, MMSE, and IADL scores and achieved a better contrast sensitivity in patients with mild $A D$ when compared to the placebo group, suggesting that EAHE is safe, well-tolerated, and may be important in achieving neurocognitive benefits.

\section{Clinical Trial Registration: ClinicalTrials.gov, identifier NCT04065061.}

Keywords: erinacine A-enriched $\boldsymbol{H}$. erinaceus mycelia, Alzheimer's disease, pilot study, prevention, magnetic resonance imaging

\section{INTRODUCTION}

The pace of population aging across the world over the past halfcentury is increasing dramatically, triggering a Silver Tsunami of chronic age-related diseases. Among these diseases, Alzheimer's Disease $(\mathrm{AD})$ is the fifth-leading cause of death among adults aged 65 years and older and is also a leading cause of disability and morbidity (Alzheimer's Association, 2019). Unlike other major diseases for which there have been steady progress in the development of novel therapies, no new pharmacologic treatment for AD has been approved since 2003 (Hung and $\mathrm{Fu}, 2017)$. One theory as to why many intervention trials have failed is that the pathophysiological process of $\mathrm{AD}$ is thought to begin many years before the onset of clinical symptoms, and the use of interventions later in the disease may not effectively slow its progression due to established pathological burden (Sperling et al., 2011). As a result, there has been a shift in the clinical research field, with the focus to develop safe and effective interventions in early and presymptomatic AD stages (Graham et al., 2017). To date, several prevention trials have been carried out and shown promising results, suggesting the potential feasibility of implementing non-pharmacological approaches, including dietary interventions (Ngandu et al., 2015; Andrieu et al., 2017).

In a recent study, the lifestyle of 633 Chinese seniors living in Singapore between 2011 and 2017 was analyzed, and it was revealed that various mushrooms have therapeutic effects in combatting $\mathrm{AD}$ by exerting neuroprotective and antioxidant effects (Feng et al., 2019). Mushrooms and their extracts have been well-known for their nutritional and culinary values, which may be regarded as novel nature-based nutraceuticals to mitigate $\mathrm{AD}$ and other age-related neurodegenerative disorders. In fact, a number of mushrooms including Hericium erinaceus (Bull.: Fr.) Pers., Dictyophora indusiata (Vent.) Desv., Grifola frondosa (Dicks.: Fr.) S.F. Gray, Tremella fuciformis Berk, Tricholoma sp., Termitomyces albuminosus (Berk.) R. Heim, Lignosus rhinocerotis (Cooke) Ryvarden, Cordyceps militaris (L.:Fr.) Link, Pleurotus giganteus (Berk.) Karunarathna and K.D. Hyde, Ganoderma lucidum P. Karst, and Ganoderma neo-japonicum Imazeki have been reported to have activities related to nerve and brain health (Phan et al., 2017). Among these, the neurohealth properties of Hericium erinaceus (Bull.:Fr.) Pers., or its common names
Lion's mane or Monkey's head mushroom, have been most extensively studied.

Hericenones and erinacines are the two important classes of constitutes isolated from the fruiting body and mycelium of H. erinaceus, respectively (Kawagishi et al., 1991, 1992, 1994, 1996a,b; Lee et al., 2000). Both hericenones and erinacines are low-molecular weight, relatively hydrophobic compounds, and proven to stimulate nerve growth factor (NGF) synthesis and promote NGF-induced neurite outgrowth in nerve cells in vitro (Lai et al., 2013). However, hericenones failed to promote NGF gene expression in $1321 \mathrm{~N} 1$ human astrocytoma cells (Mori et al., 2008) while erinacine A successfully upregulated the NGF level in the locus coeruleus and hippocampus of rats (Shimbo et al., 2005). To date, only erinacines A (unpublished results) and S (Hu et al., 2019) but not hericenones have been verified to cross the blood-brain-barrier, suggesting a greater of likelihood of them targeting the central nervous system. Furthermore, the in vivo neuroprotection of erinacine A-enriched $H$. erinaceus (EAHE) mycelia has been demonstrated in several studies against stroke, Parkinson's disease, AD, depression, and aging (Li et al., 2018b). Based on these findings, it is highly suggestive that erinacine $\mathrm{A}$ is one of the key components responsible for the neurotrophic and neuroprotective activities of $H$. erinaceus.

A previous human pilot study has been carried out to investigate the efficacy of oral administration of $H$. erinaceus with 50- to 80-year-old Japanese men and women diagnosed with mild cognitive impairment. The subjects in the $H$. erinaceus group took four $H$. erinaceus tablets three times a day for 16 weeks and showed an improvement in cognitive functions (Mori et al., 2009). However, in this study, the active constituents, representative markers, and major chemical constituents of $H$. erinaceus tablets have not been extensively addressed. While there is still a controversy regarding whether hericenones in the $H$. erinaceus fruiting body have neuroprotective activities in vivo, erinacine $\mathrm{A}$ in the $H$. erinaceus mycelia, on the other hand, confers neuroprotective effects and attenuates the oxidative stress against stroke (Lee et al., 2014), AD (Tzeng et al., 2018), Parkinson's disease (Kuo et al., 2016), depression (Chiu et al., 2018), and aging ( $\mathrm{Li}$ et al., 2019) in mouse models. As there is an urgent need to translate basic discovery research to clinical evaluation, this is the first clinical investigation of 
three $H$. erinaceus mycelia capsules $(350 \mathrm{mg} / \mathrm{capsule}$; containing $5 \mathrm{mg} / \mathrm{g}$ erinacine A active ingredient) per day for the treatment of patients with early AD.

\section{MATERIALS AND METHODS}

\section{Sample Preparation and High-Performance Liquid Chromatography (HPLC) Analysis}

Hericium erinaceus mycelia enriched with $5 \mathrm{mg} / \mathrm{g}$ erinacine A were prepared and evaluated according to a procedure described previously (Li et al., 2014b). In brief, EAHE mycelia was grown in a submerged liquid medium comprised of $0.25 \%$ yeast extract, $4.5 \%$ glucose, $0.5 \%$ soybean powder, $0.25 \%$ peptone, and 0.05 $\% \mathrm{MgSO}_{4}$ with an initial $\mathrm{pH}$ set to 4.5 at $26^{\circ} \mathrm{C}$ for 5 days. This process is then scaled up in 500-L and 20-ton fermenters for 5 days and 12 days, respectively. Following mass production, the mycelia were lyophilized, extracted with methanol, and analyzed by HPLC to quantify $5 \mathrm{mg} / \mathrm{g}$ erinacine $\mathrm{A}$ in EAHE mycelia. For study's intervention, $350 \mathrm{mg}$ EAHE mycelia were encapsulated in each gelatin capsule and used as treatments.

\section{Study Design}

The present study was a 1-year, double-blind, randomized, placebo-controlled, fixed-dose intervention pilot trial conducted at Chung Shan Medical University in patients with mild AD. The study protocol was approved by the Institutional Review Board of Chung Shan Medical University and registered with ClinicalTrials.gov under the number NCT04065061. This study comprised of a 3-week no-drug screening period, followed by a 49-week double-blind treatment period with 2-parallel groups in which eligible patients were randomized to either three $350 \mathrm{mg} /$ capsules containing $5 \mathrm{mg} / \mathrm{g}$ erinacine A per day or identical appearing placebo capsules with meals. This dose was chosen according to a previous study design (Li et al., 2019) and converted to human dose as specified by FDA guidelines (FDA, 2005). Cognitive assessments, ophthalmic examinations, biomarker collection, and neuroimaging were followed throughout the study period. Written informed consent from all patients or their legal representatives was obtained before their enrollment.

\section{Participants, Randomization, and Blinding}

The inclusion criteria for enrollment included patients with age $>50$ years and diagnosis of probable $\mathrm{AD}$ according to the Diagnostic and Statistical Manual of Mental Disorders (fourth edition, DSM-IV) (American Psychiatric Association, 2013) and National Institute of Neurological and Communicative Disorders and Stroke-Alzheimer's Disease and Related Disorders Association (Mckhann et al., 1984) criteria. The exclusion criteria included patients with severe somatic or psychiatric comorbidity as they may significantly impair cooperation with the study. Once a participant met the study's eligibility criteria, a baseline visit was planned, and thorough somatic and neurological examinations were carried out.

Following the baseline assessments, participants were randomly assigned to receive either the placebo or three EAHE mycelia capsules per day according to a randomization list produced by a computerized random-number generator. Except for two trial-independent statisticians that were unmasked, all patients, caregivers, raters, and investigators were blinded to the interventions until the database was finalized. The schedule of trial enrollment, interventions, and assessments according to the Standard Protocol Items: Recommendations for Interventional Trials (SPIRIT) Statement (Chan et al., 2013) is presented in Figure 1.

\section{Efficacy and Safety Parameters}

All participants received either three EAHE mycelia capsules per day or placebo for 49 weeks and were assessed by a rater at $0,13,25$, and 49 weeks after commencing the treatment. The efficacy of EAHE mycelia was determined by the mean change from baseline to the final analysis and was evaluated by a comprehensive battery, which included cognitive assessments, ophthalmic examinations, biomarker collection, and neuroimaging.

The cognitive assessments were performed at baseline, week 13, week 25, and week 49. Reference measures for cognition included the Neuropsychiatric Inventory (NPI) (Cummings et al., 1994), Cognitive Abilities Screening Instrument (CASI) (Teng et al., 1994), Mini-Mental State Examination (MMSE) (Folstein et al., 1975), and Instrumental Activities of Daily Living (IADL) (Nygard, 2003). These standard tests are used extensively in both clinical practice and research to measure treatment effects in patients with mild to moderate dementia.

A complete ophthalmologic examination including the measurement of best-corrected visual acuity (BCVA) and contrast sensitivity (CS) was conducted at baseline, week 25 , and week 49. Monocular and binocular best-corrected distant visual acuity were determined using a standard clinical Snellen eye chart at a 5-meter distance from the chart. The contrast sensitivity test was performed with a standard Pelli-Robson chart under the same conditions for all the patients.

Blood biomarkers were collected at baseline, week 25, and week 49. After overnight fasting, blood samples from each patient were drawn through 22-gauge needles and transferred into either ethylene diamine tetraacetic acid-potassium (EDTA-K2) tubes for hematological analysis or stored in tubes without anticoagulants for biochemical analysis. For hematological analysis, homocysteine (Hcy) and hemoglobin ( $\mathrm{Hb}$ ) were measured using an automated hematology analyzer (Gen-STM, Beckman Coulter, Inc., United States) while the serum biochemistry parameters including albumin and calcium were performed using an automated biochemistry analyzer (LX ${ }^{\circledR}-20$, Beckman Coulter, Inc., United States). Quantitative determination of other blood biomarkers such as alphal-antichymotrypsin ( $\alpha$-ACT; ab171574, Abcam, United Kingdom), amyloidbeta peptide 1-40 ( $\beta$-amyloid; CEA864Hu, Wuhan USCN Business Co., Ltd., China), apolipoprotein E4 (APOE4; K4699, BioVision Inc., United States), dehydroepiandrosterone-sulfate 


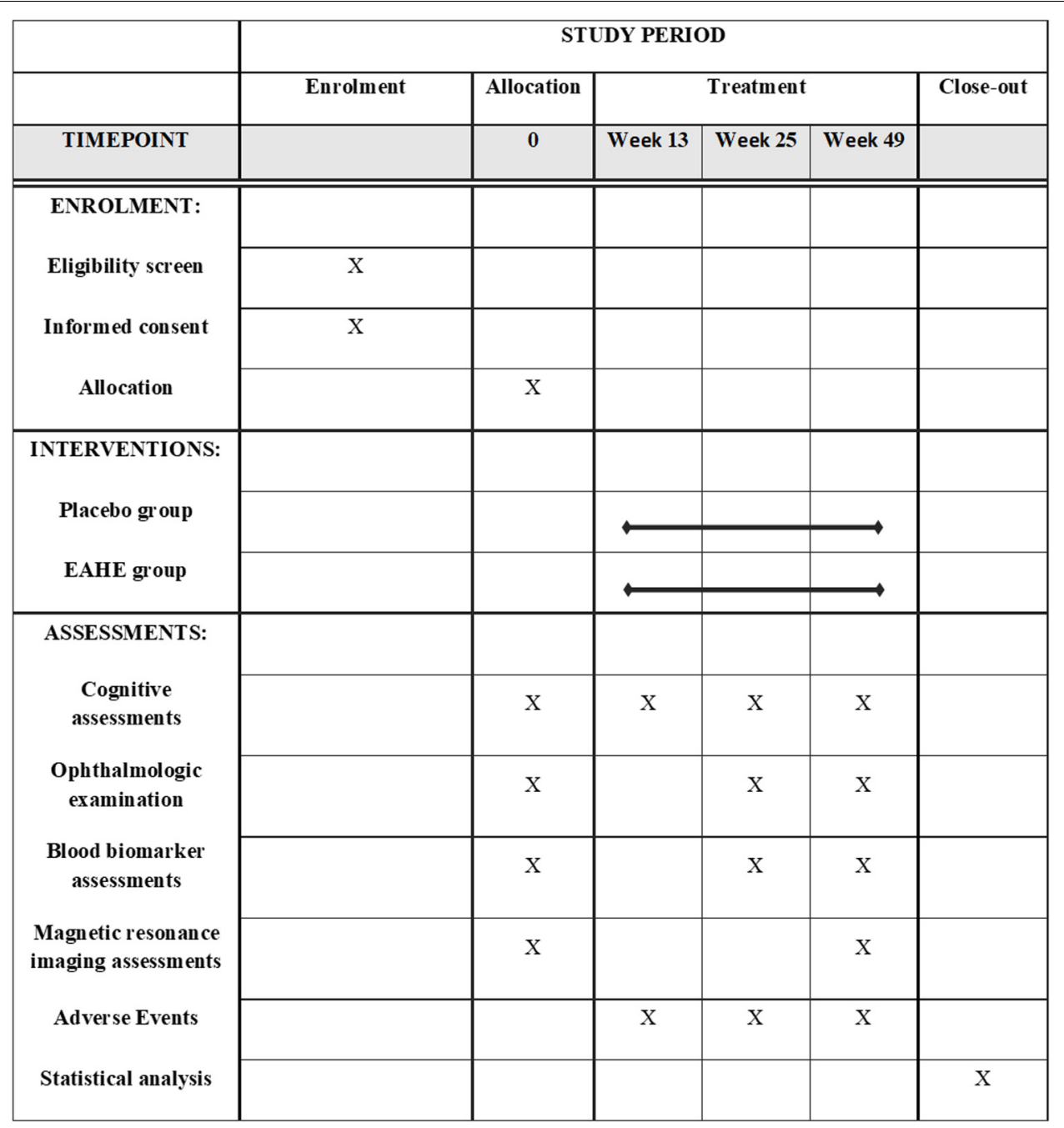

FIGURE 1 | Schedule of enrollment, interventions and assessments (SPIRIT Figure).

(DHEAS; ab108669, Abcam, United Kingdom), brain-derived neurotrophic factor (BDNF; KA0329, Abnova, Taiwan), and superoxide dismutase (SOD; \#19160, Sigma-Aldrich, United States) were measured using commercially available enzyme-linked immunosorbent assay (ELISA) kits.

Neuroimaging such as magnetic resonance imaging (MRI) assessment was performed before and after the intervention period. All subjects had brain imaging using diffusion tensor imaging (DTI) through a Siemens Magnetom Skyra threetesla (3T) scanner. Diffusion datasets were collected with the following parameters: repetition time $(\mathrm{TR})=4800 \mathrm{~ms}$, echo time $(\mathrm{TE})=97 \mathrm{~ms}$, field of view $(\mathrm{FOV})=25 \mathrm{~cm}$, image matrix $=128 \times 128$, slice number $=35$, thickness $=4 \mathrm{~mm}$, flip angle $=90^{\circ}, 4 b$-values $=0,1000,1500,2000 \mathrm{~s} / \mathrm{mm}^{2}$, diffusion direction $=64$, and bandwidth $=1562 \mathrm{~Hz} /$ pixel. The fiber number, the fractional anisotropy (FA), and the apparent diffusion coefficient (ADC) from the arcuate fasciculus (ARC), parahippocampal cingulum (PHC), inferior fronto-occipital fasciculus (IFOF), and uncinate fasciculus (UNC) regions in the dominant and non-dominant hemispheres were determined based on the diffusion tensor analyzed through using the specialized software nordicICE v4.0.2.

Safety was evaluated by monitoring adverse events according to the Food and Drug Administration regulations (Behrman Sherman et al., 2011). Moreover, adverse event reporting was also reviewed by an independent safety monitoring committee systematically throughout the study.

\section{Sample Size and Statistical Analysis}

Considering this study is a pilot study to assess the cognitive efficacy of EAHE mycelia in patients with mild $\mathrm{AD}$ and the feasibility of a further larger clinical trial, a total of at least 60 people were recruited based on a simulation study to maintain adequate power while keeping the overall sample size of the pilot and main trial together to a minimum (Teare et al., 2014). Statistical analyses were performed using SPSS software (version 18). Data are presented as means \pm standard deviation (SD). The Mann-Whitney $U$ test was used to compare the data between 


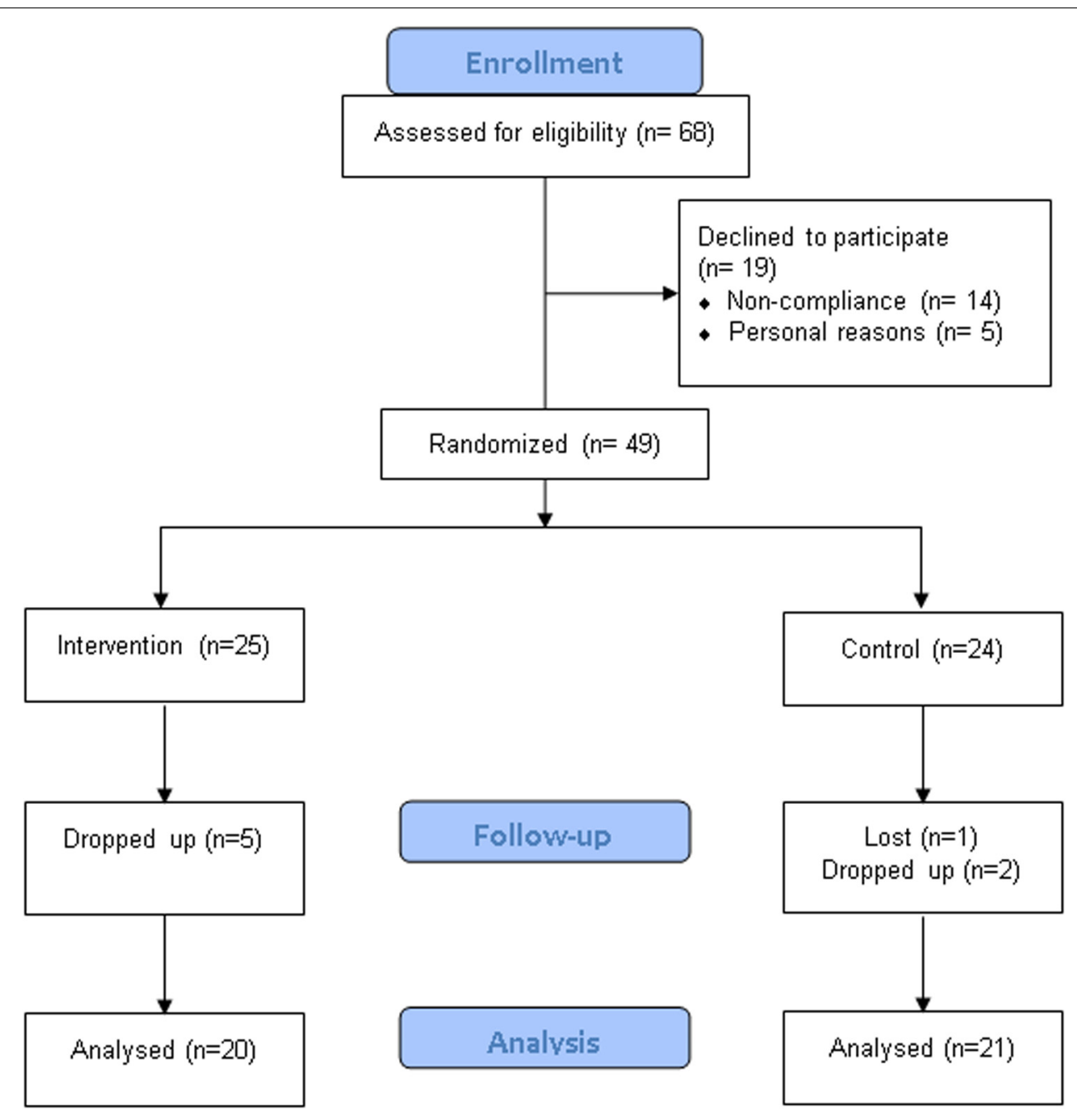

FIGURE 2 | CONSORT diagram.

the two study groups while the Wilcoxon signed-rank test was used to compare variables before and after the intervention. The statistically significant value was set at $p<0.05$.

\section{RESULTS}

\section{Participants}

A total of 68 participants who had been diagnosed with mild AD were recruited in this study. Participants were randomly assigned to either the EAHE intervention group or the placebo control group. Of the 68 patients who participated in the surveillance, 19 declined to participate due to personal reasons $(n=5)$ and noncompliance $(n=14)$. Forty-nine participants were randomized, of whom seven subjects withdrew consent and one subject lost to follow-up. In the end, 41 subjects completed the study, and the data from 17 male and 24 female study participants were analyzed (Figure 2). Demographic and baseline characteristics are shown in Table 1. There were no statistical differences between the
EAHE group and the placebo group in gender, age, and education characteristics at baseline.

\section{Cognitive Assessments}

The NPI, CASI, MMSE, and IADL tests were performed at baseline, after weeks 13, weeks 25, and weeks 49 of supplementation with EAHE. The means scores of each parameter in the EAHE and placebo group are presented in Table 2. For the NPI test, the mean NPI scores at all time-points

TABLE 1 | Participant demographics.

\begin{tabular}{lccc}
\hline Variables & $\begin{array}{c}\text { EAHE group } \\
(\boldsymbol{n}=\mathbf{2 0})\end{array}$ & $\begin{array}{c}\text { Placebo } \\
\text { group }(\boldsymbol{n}=\mathbf{2 1})\end{array}$ & $\boldsymbol{p}$-Value \\
\hline Gender (male/female) & $6 / 14$ & $11 / 10$ & - \\
Age (years) & $74.3 \pm 7.15$ & $77.05 \pm 8.2$ & 0.261 \\
Education (years) & $6.35 \pm 4.74$ & $6 \pm 5.36$ & 0.826 \\
\hline
\end{tabular}

Values are given as mean $\pm S D$. 
decreased in both the EAHE and placebo groups compared to the baseline. Although subjects who received EAHE has a lower mean NPI score than the placebo group at week $49(0.67 \pm 1.15$ vs. $2.25 \pm 3.3)$, the comparison to baseline values showed no significant difference in both groups $(p=0.077$ and 0.163 , respectively). Moreover, when compared to the baseline values and different time points of CASI scores, subjects in the EAHE group showed an increasing trend with marginal significance $(71.75 \pm 17.12$ to $75.35 \pm 15.86 ; p=0.058)$ whereas the subjects in the placebo group showed a decreasing trend from the baseline to 49 weeks $(73.52 \pm 14.51$ to $69.67 \pm 16.62 ; p=0.064)$. However, there was no significance in relation to the intra-group and inter-group CASI analysis except for the difference between the baseline and week 25 of the placebo group $(p=0.043)$. Furthermore, MMSE scores significantly increased $(21.75 \pm 6.1$ to $23.2 \pm 5.92 ; p=0.035$ ) in the EAHE group from the baseline to week 49 whereas the comparison of all time-points showed no significant change in the placebo group. Nevertheless, all the pairwise comparisons of the MMSE test were not statistically significant $(p>0.05)$. Finally, for the IADL test, no baseline differences between the EAHE and placebo groups at any time points were observed except for the pairwise comparison at week 49 which was statistically significant $(6.7 \pm 2.47$ vs. $5 \pm 2.7$; $p=0.012$, respectively).

\section{Ophthalmologic Examination}

Table 3 summarizes the ophthalmologic examination for the EAHE and placebo groups after 25 and 49 weeks of intervention. The analysis of BCVA in OD (right eye), OS (left eye), and OU (both eyes) of the EAHE and placebo groups showed no difference from their baselines to the end of the study. Although significant baseline differences in BCVA of OD, OS, and OU were found between the groups, these differences remained unchanged throughout the study period except for BCVA OS at week 25 $(p=0.101)$. Meanwhile, subjects with EAHE treatment showed improvements in the mean monocular CS (OD: $0.84 \pm 0.19$ to $0.90 \pm 0.08$; OS: $0.83 \pm 0.2$ to $0.86 \pm 0.13)$ and binocular CS (OU: $0.88 \pm 0.15$ to $0.89 \pm 0.11$ ) values following 49 weeks of intervention whereas the placebo group showed an upward trend in the CS OD $(0.72 \pm 0.32$ to $0.77 \pm 0.27)$, a downward trend in the CS OS $(0.83 \pm 0.18$ to $0.78 \pm 0.17)$, and remained unchanged $(0.85 \pm 0.19$ to $0.85 \pm 0.08)$ in the CS OU at the end of the study. Nevertheless, all these groups did not reach statistical significance except for the changes in CS OU from baseline to 49 weeks in the placebo group $(0.85 \pm 0.19$ to $0.85 \pm 0.08$; $p=0.033)$ and differences of CS OS at week 49 between treatment groups $(p=0.046)$.

\section{Blood Biomarker Assessments}

With further analysis of the blood biomarkers over the 49week study period within the groups (Table 4), significant improvements of Hcy at week 25 and 49 ( $p=0.007$ and $p=0.012$, respectively) were observed in the EAHE group while significant negative effects in calcium at week $25(p=0.004)$, albumin at week $49(p=0.004), \mathrm{Hb}$ at week 25 and $49(p=0.003$ and $p=0.009$, respectively), and BDNF at week $25(p=0.012)$ were noted in the placebo group. Moreover, although both groups showed significant decreases in SOD and APOE4 as well as significant increases in $\alpha$-ACT and $\beta$-amyloid ( $p<0.05$ ), APOE4, $\alpha$-ACT, and $\beta$-amyloid had an improving trend in the EAHE group than the placebo group at week 49. No significant difference, however, was observed for all other parameters.

\section{Magnetic Resonance Imaging (MRI) Assessments}

The total fiber number, FA, and ADC values from the ARC, $\mathrm{PHC}$, IFOF, and UNC regions in the dominant and nondominant hemispheres of the EAHE and control group are listed in Table 5. After 49 weeks of EAHE intervention, the total fibers were significantly less decreased than those in the placebo group. Nevertheless, they did not reach statistical significance between groups $(p=0.715)$. In addition, compared to their baselines, the mean ADC values from the ARC region in the dominant hemisphere significantly increased in the placebo group while the $\mathrm{ADC}$ values from the $\mathrm{PHC}$ region in the dominant hemisphere significantly decreased in the EAHE group at week 49 . No statistically significant differences were found in other parameters.

\section{Adverse Events}

During the study, 1 subject lost to follow-up while 7 subjects $(7 / 49 ; 14.3 \%)$ left the study. Reasons for dropout that have been investigated include unsatisfactory efficacy ( 2 from EAHE group and 1 from the placebo group) and the presence of side effects ( 3 from EAHE group and 1 from the placebo group). Possible or probable side effects related to the intervention included nausea in the placebo group and abdominal discomfort, nausea, and skin rash in the EAHE group.

\section{DISCUSSION}

Diet is an important modifiable risk factor for AD (Sindi et al., 2018) as it is able to modulate structural brain connectivity (Park et al., 2018), cause positive changes in brain function and behavior (Bolton and Bilbo, 2014), as well as help regulate cognition and emotion (Spencer et al., 2017). As benefits of EAHE associated with brain and nerve health have been wellstudied ( $\mathrm{Li}$ et al., 2018b), this is the first study to endorse its potential in mitigating neurodegenerative disorders. Based on the results of this pilot, randomized, double-blinded, controlled study, subjects with mild AD showed a significant benefit in reducing cognitive decline and improving contrast sensitivity after oral administration of three $5 \mathrm{mg} / \mathrm{g}$ EAHE mycelia capsules per day for 49 weeks when compared with placebo.

In this study, through random allocation, the baseline demographic information including age, gender, and education level between EAHE and placebo groups showed no significant differences before the intervention. Nevertheless, after the intervention, a significant deterioration in CASI from baseline to week 25 was noted in the placebo group, a significant improvement in MMSE from baseline to week 49 was observed in the EAHE group, and a significant IADL difference at week 49 were found between the two groups. Higher CASI and 
TABLE 2 | Comparison of cognitive assessments between EAHE and placebo groups.

\begin{tabular}{|c|c|c|c|c|c|}
\hline \multirow[b]{2}{*}{ Variables } & \multicolumn{2}{|c|}{ EAHE group $(n=20)$} & \multicolumn{2}{|c|}{ Placebo group $(n=21)$} & \multirow[b]{2}{*}{ Intergroup $p$-value } \\
\hline & Value & Intragroup $p$-value & Value & Intragroup $p$-value & \\
\hline \multicolumn{6}{|l|}{ NPI } \\
\hline Baseline & $4.21 \pm 6.62$ & - & $3 \pm 5.2$ & - & 0.696 \\
\hline Week 13 & $0 \pm 0$ & 0.306 & $1.33 \pm 1.53$ & 0.812 & 0.259 \\
\hline Week 25 & $1.5 \pm 2.12$ & 0.439 & $1.33 \pm 2.31$ & 0.087 & 0.989 \\
\hline Week 49 & $0.67 \pm 1.15$ & 0.077 & $2.25 \pm 3.3$ & 0.163 & 0.129 \\
\hline \multicolumn{6}{|l|}{ CASI } \\
\hline Baseline & $71.75 \pm 17.12$ & - & $73.52 \pm 14.51$ & - & 0.804 \\
\hline Week 13 & $72.74 \pm 15.83$ & 0.246 & $70.71 \pm 19.3$ & 0.13 & 0.881 \\
\hline Week 25 & $73.8 \pm 17.15$ & 0.313 & $69.62 \pm 16.07$ & $0.043^{*}$ & 0.368 \\
\hline Week 49 & $75.35 \pm 15.86$ & 0.058 & $69.67 \pm 16.62$ & 0.064 & 0.315 \\
\hline \multicolumn{6}{|l|}{ MMSE } \\
\hline Baseline & $21.75 \pm 6.1$ & - & $21.33 \pm 5.74$ & - & 0.629 \\
\hline Week 13 & $22.58 \pm 5.6$ & 0.065 & $20.95 \pm 6.47$ & 0.616 & 0.4 \\
\hline Week 25 & $22.55 \pm 6.24$ & 0.23 & $21.05 \pm 5.5$ & 0.686 & 0.353 \\
\hline Week 49 & $23.2 \pm 5.92$ & $0.035^{*}$ & $20.67 \pm 6.17$ & 0.661 & 0.261 \\
\hline \multicolumn{6}{|l|}{ IADL } \\
\hline Baseline & $6.35 \pm 2.81$ & - & $5.71 \pm 2.65$ & - & 0.25 \\
\hline Week 13 & $6.37 \pm 2.31$ & 1 & $6 \pm 2.07$ & 0.484 & 0.423 \\
\hline Week 25 & $6.45 \pm 2.58$ & 0.705 & $5.57 \pm 2.29$ & 0.634 & 0.11 \\
\hline Week 49 & $6.7 \pm 2.47$ & 0.157 & $5 \pm 2.7$ & 0.075 & $0.012^{*}$ \\
\hline
\end{tabular}

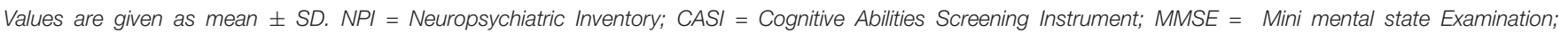
$I A D L=$ Instrumental Activities of Daily Living. ${ }^{*} p<0.05$.

MMSE scores represent better cognition, and higher IADL scores represent a lower level of dependence (Chiu et al., 2016). Although there were no significant differences in CASI and MMSE between the EAHE and placebo groups, the scores were higher in the EAHE group compared to those in the placebo group for participants with mild $\mathrm{AD}$, implying that subjects could achieve more benefits from the intervention.

To date, human studies on $H$. erinaceus are scarce. Only three trials were found to examine the efficacy of oral administration of $H$. erinacues for improving brain pathology. In one doubleblind placebo-controlled study, 50- to 80-year-old Japanese men and women $(n=30)$ diagnosed with mild cognitive impairment showed marked improvement in cognitive function when compared to controls, using a cognitive function scale based on the revised Hasegawa Dementia Scale and following the effects of oral intake of four $250 \mathrm{mg}$ tablets containing $96 \%$ of $H$. erinaceus fruiting body dry powder three times a day for 16 weeks (Mori et al., 2009). In another randomized, double-blind placebo-controlled study, administration of $0.5 \mathrm{~g}$ $H$. erinaceus fruiting body in cookies over 4 weeks showed a reduction in anxiety and depression in menopausal women ( $n=30)$ compared to those taking placebo, as measured by the Center for Epidemiologic Studies Depression Scale and Indefinite Complaints Index (Nagano et al., 2010). In the third randomized, double-blind, placebo-controlled parallelgroup comparative study, the consumption of cookies containing $0.8 \mathrm{~g}$ of $\mathrm{H}$. erinaceus fruiting body dry powder alleviated the deterioration of short memories and improved the cognitive functions in 31 participants with an average age of 61.3 years old over the period of 12 weeks, as measured by MMSE (Saitsu et al., 2019). Prior studies have reported that NGF could enhance neurogenesis-inducing effects, which led to antidepressant and antianxiety activities (Shohayeb et al., 2018). Although hericenones C and D from the fruiting body of $H$. erinaceus have shown to induce neuroprotective properties (Kawagishi et al., 1991) in rats by stimulating NGF synthesis via activation of the c-jun $\mathrm{N}$-terminal kinase (JNK) pathway, they failed to promote NGF gene expression in 1321N1 human astrocytoma cells (Mori et al., 2008). This result suggested that $H$. erinaceus fruiting body may contains other active compounds and/or hericenones that can potentially improve mild cognitive impairment as well as reduce depression and anxiety.

On the contrary, erinacines isolated from the mycelium of the mushroom are able to pass through the brain-blood barrier into the brain ( $\mathrm{Hu}$ et al., 2019) to promote NGF synthesis in vivo (Shimbo et al., 2005). During normal physiological conditions, NGF is released by the postsynaptic cortical and hippocampal neurons to activate further signaling cascades that include cell survival, maintenance, and proliferation (Biane et al., 2014). However, NGF has been found to be reduced during the pathological conditions of $\mathrm{AD}$, resulting in induced loss of cortical synapses and atrophy of cholinergic neurons in the basal forebrain (Iulita and Cuello, 2014). Moreover, analyzing AD11 anti-NGF transgenic mice that express NGF antibodies in the brain, it was observed that NGF deprivation leads to early inflammation and Alzheimer's neurodegeneration (Capsoni et al., 2011). In this regard, as erinacine $A$ has been proven to promote NGF synthesis in vivo, it may contribute to the 
TABLE 3 | Comparison of ophthalmologic examination between EAHE and placebo groups.

\begin{tabular}{|c|c|c|c|c|c|}
\hline \multirow[b]{2}{*}{ Variables } & \multicolumn{2}{|c|}{ EAHE group $(n=20)$} & \multicolumn{2}{|c|}{ Placebo group $(n=21)$} & \multirow[b]{2}{*}{ Intergroup $p$-value } \\
\hline & Value & Intragroup $p$-value & Value & Intragroup $p$-value & \\
\hline Baseline & $0.83 \pm 0.24$ & - & $0.59 \pm 0.33$ & - & $0.013^{\star}$ \\
\hline Week 25 & $0.8 \pm 0.27$ & 0.552 & $0.59 \pm 0.33$ & 0.814 & $0.026^{\star}$ \\
\hline Week 49 & $0.83 \pm 0.2$ & 0.545 & $0.57 \pm 0.28$ & 0.258 & $0.005^{\star}$ \\
\hline Baseline & $0.82 \pm 0.25$ & - & $0.63 \pm 0.27$ & - & $0.024^{*}$ \\
\hline Week 25 & $0.77 \pm 0.27$ & 0.153 & $0.67 \pm 0.23$ & 0.633 & 0.101 \\
\hline Week 49 & $0.82 \pm 0.24$ & 0.824 & $0.64 \pm 0.23$ & 0.201 & $0.025^{\star}$ \\
\hline \multicolumn{6}{|l|}{ BCVA OU } \\
\hline Baseline & $0.9 \pm 0.22$ & - & $0.75 \pm 0.23$ & - & $0.017^{\star}$ \\
\hline Week 25 & $0.86 \pm 0.19$ & 0.257 & $0.75 \pm 0.29$ & 0.187 & 0.212 \\
\hline Week 49 & $0.9 \pm 0.08$ & 0.257 & $0.77 \pm 0.27$ & 0.582 & 0.089 \\
\hline \multicolumn{6}{|l|}{ cs Os } \\
\hline Baseline & $0.83 \pm 0.2$ & - & $0.83 \pm 0.18$ & - & 0.323 \\
\hline Week 25 & $0.82 \pm 0.25$ & 0.85 & $0.82 \pm 0.11$ & 0.227 & 0.155 \\
\hline Week 49 & $0.86 \pm 0.13$ & 0.606 & $0.78 \pm 0.17$ & 0.13 & $0.046^{\star}$ \\
\hline \multicolumn{6}{|l|}{ CSOU } \\
\hline Baseline & $0.88 \pm 0.15$ & - & $0.85 \pm 0.19$ & - & 0.28 \\
\hline Week 25 & $0.88 \pm 0.2$ & 0.739 & $0.86 \pm 0.09$ & 0.405 & 0.069 \\
\hline Week 49 & $0.89 \pm 0.11$ & 0.68 & $0.85 \pm 0.08$ & $0.033^{*}$ & 0.056 \\
\hline
\end{tabular}

Values are given as mean $\pm S D$. BCVA = best-corrected visual acuity; $C S=$ contrast sensitivity; $O D=$ oculus dexter; $O S=0 c u l u s$ sinister; $O U=0 c u l u s$ uterque. ${ }^{*} p<0.05$.

survival and regeneration of cholinergic neurons as well as revive cholinergic signaling in the cortex and hippocampus, thereby improving the cognitive ability in subjects with mild AD. However, the precise mechanism of its action needs further investigation.

Not only could NGF markedly protect degenerating neurons in the brain, studies have also shown that NGF administration could modulate the development and differentiation of the retina and the optic nerve, as well as promote the survival and recovery of retinal ganglion cells (Aloe et al., 2012). To our knowledge, this is the first study to examine the efficacy of EAHE on visual acuity and contrast sensitivity. No significant differences were observed in the ophthalmologic examination in this study except for a higher CS OS at week 49 after EAHE treatment. This finding of EAHE as a NGF stimulator in improving $\mathrm{CS}$ but not VA in subjects with mild $\mathrm{AD}$ is consistent with previous studies. They have found that contrast sensitivity was significantly reduced in patients with $\mathrm{AD}$ compared to elderly control subjects while no significant difference in visual acuity were found between the patients with $\mathrm{AD}$ and control subjects (Crow et al., 2003) suggesting that EAHE targeting astrocytes only responded to an injury or damaged area by maintaining neurogenesis as a mechanism of repair (Poulose et al., 2017). Future studies, however, are required to further explore this possible mechanism.
With EAHE consumption, it is important to note that an altered diet or a multiplicity of environmental changes could change the blood proteome as well as ions (Te Pas et al., 2013). In different studies, EAHE treatment was accompanied by improvements in blood biomarkers in subjects with mild AD. Biomarkers monitoring based on biochemical analysis of blood during or after the intervention period could offer considerable promise for improving the treatment of AD (Cummings et al., 2019). Recent studies have identified a biomarker panel that included blood-based markers that significantly increased alpha-1-Antichymotrypsin, $\beta$-amyloid, superoxide dismutase, and homocysteine levels as well as decreased calcium, albumin, dehydroepiandrosterone sulfate, apolipoprotein E, hemoglobin, and BDNF levels in AD (Dekosky et al., 2003; Laske et al., 2011; Doecke et al., 2012; Ng et al., 2019; Pan et al., 2019). Consistent with the current study, biomarkers such as calcium, albumin, APOE4, Hb, and BDNF were significantly lowered while $\alpha$-ACT and $\beta$ - amyloid were significantly elevated during the study period in the placebo group. However, there were no significant changes in calcium, albumin, $\mathrm{Hb}$, and $\mathrm{BDNF}$ compared to the baseline and a trend toward improving SOD, APOE4, and $\alpha$-ACT levels were observed in the EAHE group, indicating that EAHE may have possible effects in arresting or delaying further neurodegenerative processes. 
TABLE 4 | Comparison of blood biomarkers between EAHE and placebo groups.

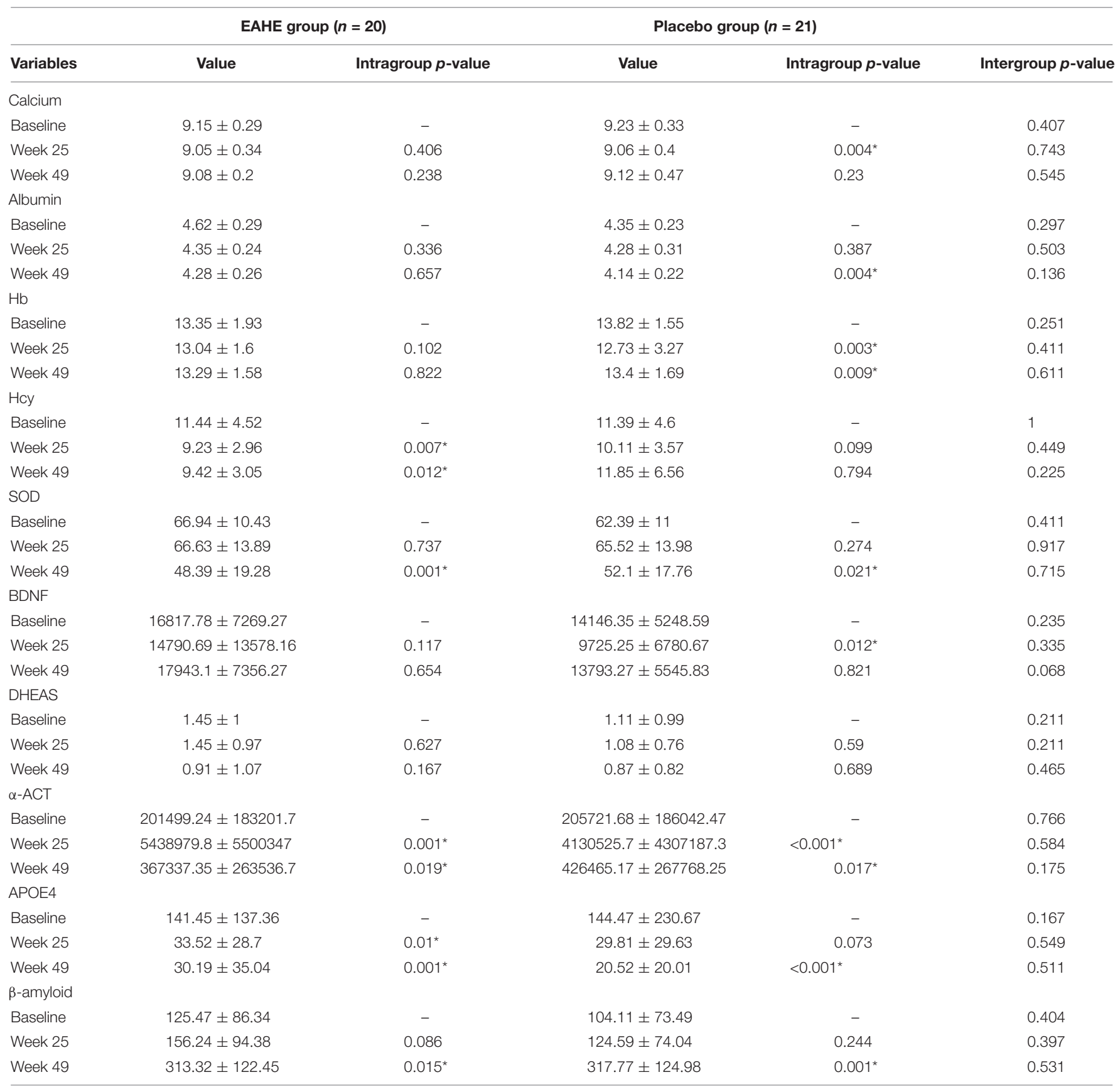

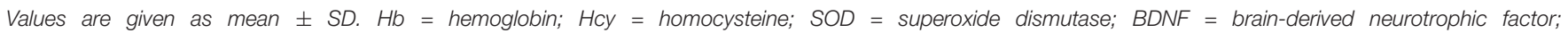
$D H E A S=$ dehydroepiandrosterone-sulphate; $\alpha-A C T=$ alpha 1-antichymotrypsin; APOE4 = apolipoprotein E4; $\beta$-amyloid = amyloid-beta peptide 1-40. ${ }^{*} p<0.05$.

The effects of EAHE on the rate of neurodegeneration could also be detected using advanced MRI, such as DTI, to probe human brain microstructures (Cho et al., 2008). DTI provides quantitative measures of $\mathrm{FA}$ and $\mathrm{ADC}$, which enable the assessment of the cellular microstructure and fiber tract integrity in live tissues. Fiber tracts such as PHC and UNC contribute to learning and memory, and IFOF and AF contribute to language functioning (Mcdonald et al., 2008). These were evaluated in each subject to depict their global white matter status. In this study, although a statistical significance was not found between the groups, the total fibers of six fiber tracts calculated were significantly less decreased than those in the placebo group after EAHE intervention, suggesting that EAHE ameliorates the loss of fiber numbers by stimulating NGF synthesis and inducing neurogenesis. Moreover, within the six fiber tracts, the mean $\mathrm{ADC}$ values from the ARC region in the dominant hemisphere significantly increased in the placebo group while no significant difference was found in the EAHE group in comparison to their 
TABLE 5 | Comparison of MRI assessments between EAHE and placebo groups.

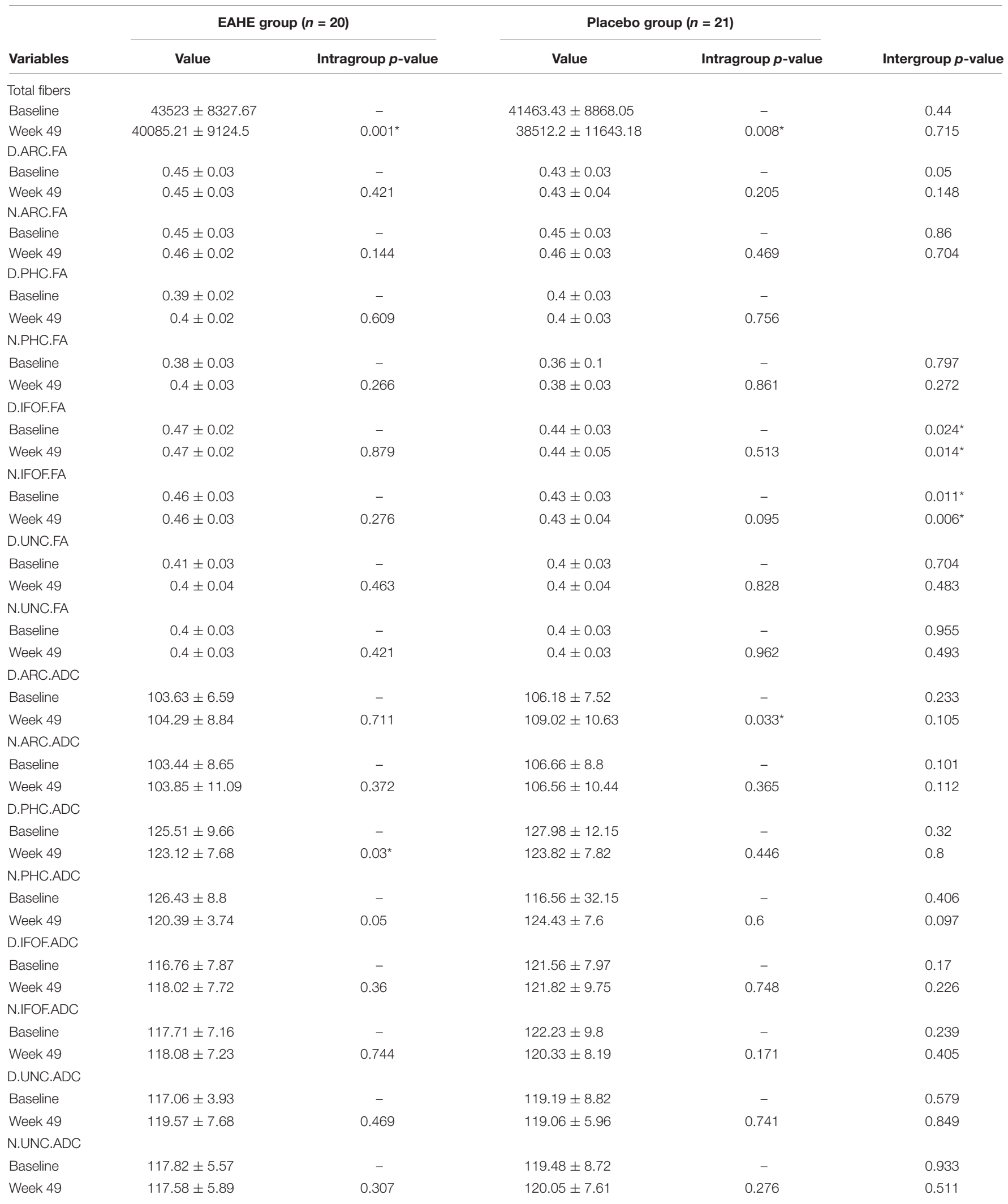

Values are given as mean $\pm S D . D=$ dominant; $N=$ Non-dominant; $A R C=$ arcuate fasciculus; $P H C=$ parahippocampal cingulum; IFOF = inferior fronto-occipital fasciculus; UNC = uncinate fasciculus; $F A=$ Fractional Anisotropy; $A D C=$ apparent diffusion coefficient. ${ }^{*} p<0.05$. 
baselines, implying that there was a greater disorganization in the neural structure observed in the placebo group. Moreover, $\mathrm{ADC}$ values from the $\mathrm{PHC}$ region in the dominant hemisphere significantly decreased in the EAHE group whereas no significant difference was found in the placebo group when compared to their baselines, indicating that there was a more well-organized neural structure noted in the EAHE group. These results tie well with a previous study wherein the ADC values increased in the ARC and $\mathrm{PHC}$ of patients with mild $\mathrm{AD}$ when compared with a control group (Mayo et al., 2019), signifying that EAHE could improve structural deterioration of ARC and $\mathrm{PHC}$ in patients with mild AD.

Lastly, despite four subjects who dropped out during the study period due to reported adverse events such as abdominal discomfort, nausea, and skin rash, no other adverse event were reported. The overall incidence was $8.2 \%$ during the entire 49 weeks. However, due to the increasing trend in clinical practice to treat elderly patients with multiple medications (Poleksic and Xie, 2019), it remains a challenge to identify if these adverse events were caused by EAHE consumption. Yet, reports on genotoxicity (Li et al., 2014a), acute toxicity (Li et al., 2018a), 28 days subchronic toxicity (Li et al., 2014b) 90 days subchronic toxicity (Lee et al., 2019) and teratotoxicity (Li et al., 2018a) have been conducted in animals and showed no adverse effects. Moreover, no adverse events have been reported after the launch of EAHE products into the Taiwanese market since 2015 (Li et al., 2018b). Nevertheless, further studies, especially the serum biochemical and hematological data along with urinalysis values after longterm consumption in humans, are important to consider.

In comparison to the placebo group, the intake of EAHE for 49 weeks showed higher CASI, MMSE, and IADL scores and achieved a better contrast sensitivity in patients with mild $\mathrm{AD}$. The benefit of EAHE in reducing cognitive decline may be associated with improved blood biomarkers such as calcium,

\section{REFERENCES}

Aloe, L., Rocco, M. L., Bianchi, P., and Manni, L. (2012). Nerve growth factor: from the early discoveries to the potential clinical use. J. Transl. Med. 10:239. doi: 10.1186/1479-5876-10-239

Alzheimer's Association, (2019). 2019 Alzheimer's disease facts and figures. Alzheimer Dement. 15, 321-387.

American Psychiatric Association, (2013). Diagnostic and Statistical Manual of Mental Disorders: DSM-5, 5th Edn, Arlington, VA: American Psychiatric Publishing Inc.

Andrieu, S., Guyonnet, S., Coley, N., Cantet, C., Bonnefoy, M., Bordes, S., et al. (2017). Effect of long-term omega 3 polyunsaturated fatty acid supplementation with or without multidomain intervention on cognitive function in elderly adults with memory complaints (MAPT): a randomised, placebo-controlled trial. Lancet Neurol. 16, 377-389. doi: 10.1016/S1474-4422(17)30040-6

Behrman Sherman, R., Woodcock, J., Norden, J., Grandinetti, C., and Temple, R. J. (2011). New FDA regulation to improve safety reporting in clinical trials. N. Engl. J. Med. 365, 3-5. doi: 10.1056/NEJMp1103464

Biane, J., Conner, J. M., and Tuszynski, M. H. (2014). Nerve growth factor is primarily produced by GABAergic neurons of the adult rat cortex. Front. Cell Neurosci. 8:220. doi: 10.3389/fncel.2014.00220

Bolton, J. L., and Bilbo, S. D. (2014). Developmental programming of brain and behavior by perinatal diet: focus on inflammatory mechanisms. Dialog. Clin. Neurosci. 16, 307-320. albumin, Hb, Hcy, SOD, BDNF, APOE4, and $\alpha$-ACT, as well as reduced structural deterioration in the $\mathrm{ARC}$ and $\mathrm{PHC}$ regions of patients with mild AD. However, further studies on the mechanism of action of EAHE at the biochemical and molecular levels are necessary. In addition, although EAHE is safe and welltolerated, a larger study is required to determine the benefits of EAHE consumption for patients with MCI or mild AD.

\section{DATA AVAILABILITY STATEMENT}

All datasets generated for this study are included in the article/supplementary material.

\section{ETHICS STATEMENT}

The study protocol was approved by the Institutional Review Board of the Chung Shan Medical University and registered with ClinicalTrials.gov under the number NCT04065061. The patients/participants provided their written informed consent to participate in this study.

\section{AUTHOR CONTRIBUTIONS}

I-CL analyzed the data and wrote the manuscript. H-HC, C-HL, and T-HL conceived and performed the experiments. W-PC, L-YL, Y-WC, and Y-PC provided the reagents. C-CC and DP-CL provided the expertise and feedback.

\section{ACKNOWLEDGMENTS}

The authors thank Hsin Yun Yang for editing the manuscript.

Capsoni, S., Brandi, R., Arisi, I., D'onofrio, M., and Cattaneo, A. (2011). A dual mechanism linking NGF/proNGF imbalance and early inflammation to Alzheimer's disease neurodegeneration in the AD11 anti-NGF mouse model. CNS Neurol. Disord. Drug Targets 10, 635-647. doi: 10.2174/ 187152711796235032

Chan, A. W., Tetzlaff, J. M., Altman, D. G., Laupacis, A., Gøtzsche, P. C., KrležaJeriæ, K., et al. (2013). SPIRIT 2013 statement: defining standard protocol items for clinical trials. Ann. Intern. Med. 158, 200-207.

Chiu, C.-H., Chyau, C.-C., Chen, C.-C., Lee, L.-Y., Chen, W.-P., Liu, J.-L., et al. (2018). Erinacine A-Enriched Hericium erinaceus mycelium produces antidepressant-like effects through modulating BDNF/PI3K/Akt/GSK-3 $\beta$ signaling in mice. Intern. J. Mol. Sci. 19:341. doi: 10.3390/ijms1902 0341

Chiu, P.-Y., Tsai, C.-T., Chen, P., Chen, W.-J., and Lai, T.-J. (2016). Neuropsychiatric symptoms in parkinson's disease dementia are more similar to alzheimer's disease than dementia with lewy bodies: a case-control study. PLoS One 11:e0153989. doi: 10.1371/journal.pone.0153989

Cho, H., Yang, D. W., Shon, Y. M., Kim, B. S., Kim, Y. I., Choi, Y. B., et al. (2008). Abnormal integrity of corticocortical tracts in mild cognitive impairment: a diffusion tensor imaging study. J. Korea. Med. Sci. 23, 477-483. doi: 10.3346/ jkms.2008.23.3.477

Crow, R. W., Levin, L. B., Labree, L., Rubin, R., and Feldon, S. E. (2003). Sweep visual evoked potential evaluation of contrast sensitivity in Alzheimer's dementia. Invest. Ophthalmol. Vis. Sci. 44, 875-878. doi: 10.1167/iovs.01-1101 
Cummings, J., Feldman, H. H., and Scheltens, P. (2019). The "rights" of precision drug development for Alzheimer's disease. Alzheimer Res. Therapy 11:76. doi: 10.1186/s13195-019-0529-5

Cummings, J. L., Mega, M., Gray, K., Rosenberg-Thompson, S., Carusi, D. A., and Gornbein, J. (1994). The neuropsychiatric inventory: comprehensive assessment of psychopathology in dementia. Neurology 44, 2308-2314. doi: 10.1212/wnl.44.12.2308

Dekosky, S. T., Ikonomovic, M. D., Wang, X., Farlow, M., Wisniewski, S., Lopez, J., et al. (2003). Plasma and cerebrospinal fluid alpha1-antichymotrypsin levels in Alzheimer's disease: correlation with cognitive impairment. Ann. Neurol. 53, 81-90. doi: 10.1002/ana.10414

Doecke, J. D., Laws, S. M., Faux, N. G., Wilson, W., Burnham, S. C., Lam, C.P., et al. (2012). Blood-based protein biomarkers for diagnosis of Alzheimer disease. Arch. Neurol. 69, 1318-1325. doi: 10.1001/archneurol.2012.1282

FDA, (2005). Estimating the Maximum Safe Starting Dose in Initial Clinical Trials for Therapeutics in Adult Healthy Volunteers. Available: https: //www.fda.gov/regulatory-information/search-fda-guidance-documents/ estimating-maximum-safe-starting-dose-initial-clinical-trials-therapeuticsadult-healthy-volunteers (accessed June 26, 2018).

Feng, L., Cheah, I. K., Ng, M. M., Li, J., Chan, S. M., Lim, S. L., et al. (2019). The association between mushroom consumption and mild cognitive impairment: a community-based cross-sectional study in singapore. J. Alzheimers Dis. 68, 197-203. doi: 10.3233/JAD- 180959

Folstein, M. F., Folstein, S. E., and Mchugh, P. R. (1975). Mini-mental state. A practical method for grading the cognitive state of patients for the clinician. J. Psychiatr. Res. 12, 189-198. doi: 10.1016/0022-3956(75)90026-6

Graham, W. V., Bonito-Oliva, A., and Sakmar, T. P. (2017). Update on Alzheimer's disease therapy and prevention strategies. Science 68, 413-430. doi: 10.1146/ annurev-med-042915-103753

Hu, J.-H., Li, I.-C., Lin, T.-W., Chen, W.-P., Lee, L.-Y., Chen, C.-C., et al. (2019). Absolute bioavailability, tissue distribution, and excretion of erinacine $\mathrm{s}$ in Hericium erinaceus Mycelia. Cell 24:1624. doi: 10.3390/molecules24081624

Hung, S.-Y., and Fu, W.-M. (2017). Drug candidates in clinical trials for Alzheimer's disease. J. Biomed. Sci. 24:47. doi: 10.1039/c7an02013a

Iulita, M. F., and Cuello, A. C. (2014). Nerve growth factor metabolic dysfunction in Alzheimer's disease and Down syndrome. Trends Pharmacol. Sci. 35, 338348. doi: 10.1016/j.tips.2014.04.010

Kawagishi, H., Ando, M., Sakamoto, H., Yoshida, S., Ojima, F., Ishiguro, Y., et al. (1991). Hericenones C, D and E, stimulators of nerve growth factor (NGF)-synthesis, from the mushroom Hericium erinaceum. Tetrahed. Lett. 32, 4561-4564.

Kawagishi, H., Ando, M., Shinba, K., Sakamoto, H., Yoshida, S., Ojima, F., et al. (1992). Chromans, hericenones F, G and H from the mushroom Hericium erinaceum. Phytochemistry 32, 175-178.

Kawagishi, H., Shimada, A., Hosokawa, S., Mori, H., Sakamoto, H., Ishiguro, Y., et al. (1996a). Erinacines E, F, and G, stimulators of nerve growth factor (NGF)-synthesis, from the mycelia of Hericium erinaceum. Tetrahed. Lett. 37, 7399-7402.

Kawagishi, H., Simada, A., Shizuki, K., Ojima, F., Mori, H., Okamoto, K., et al. (1996b). Erinacine D, a stimulator of ngf-synthesis, from the mycelia of Hericium Erinaceum. Heterocyc. Commun. 2, 51-54. doi: 10.1515/HC.1996. 2.1.51

Kawagishi, H., Shimada, A., Shirai, R., Okamoto, K., Ojima, F., Sakamoto, H., et al. (1994). Erinacines A, B and C, strong stimulators of nerve growth factor (NGF)-synthesis, from the mycelia of Hericium erinaceum. Tetrahed. Lett. 35, 1569-1572.

Kuo, H. C., Lu, C. C., Shen, C. H., Tung, S. Y., Hsieh, M. C., Lee, K. C., et al. (2016). Hericium erinaceus mycelium and its isolated erinacine A protection from MPTP-induced neurotoxicity through the ER stress, triggering an apoptosis cascade. J. Transl. Med. 14:78. doi: 10.1186/s12967-016-0831-y

Lai, P. L., Naidu, M., Sabaratnam, V., Wong, K. H., David, R. P., Kuppusamy, U. R., et al. (2013). Neurotrophic properties of the Lion's mane medicinal mushroom, Hericium erinaceus (higher basidiomycetes) from Malaysia. Int. J. Med. Mushrooms 15, 539-554. doi: 10.1615/intjmedmushr.v15.i6.30

Laske, C., Leyhe, T., Stransky, E., Hoffmann, N., Fallgatter, A. J., and Dietzsch, J. (2011). Identification of a blood-based biomarker panel for classification of Alzheimer's disease. Intern. J. Neuropsychopharmacol. 14, 1147-1155. doi: $10.1017 /$ S1461145711000459
Lee, E. W., Shizuki, K., Hosokawa, S., Suzuki, M., Suganuma, H., Inakuma, T., et al. (2000). Two novel diterpenoids, erinacines $\mathrm{H}$ and I from the mycelia of Hericium erinaceum. Biosci. Biotechnol. Biochem. 64, 2402-2405. doi: 10.1271/ bbb.64.2402

Lee, K. F., Chen, J. H., Teng, C. C., Shen, C. H., Hsieh, M. C., Lu, C. C., et al. (2014). Protective effects of Hericium erinaceus mycelium and its isolated erinacine A against ischemia-injury-induced neuronal cell death via the inhibition of iNOS/p38 MAPK and nitrotyrosine. Int. J. Mol. Sci. 15, 15073-15089. doi: 10.3390/ijms 150915073

Lee, L.-Y., Li, I.-C., Chen, W.-P., Tsai, Y.-T., Chen, C.-C., and Tung, K.-C. (2019). Thirteen-week oral toxicity evaluation of erinacine A enriched lion's mane medicinal mushroom, Hericium erinaceus (Agaricomycetes), mycelia in sprague-dawley rats. Intern. J. Med. Mushrooms 21, 401-411. doi: 10.1615/ IntJMedMushrooms.2019030320

Li, I. C., Chen, W. P., Chen, Y. P., Lee, L. Y., Tsai, Y. T., and Chen, C. C. (2018a). Acute and developmental toxicity assessment of erincine A-enriched Hericium erinaceus mycelia in Sprague-Dawley rats. Drug Chem. Toxicol. 41, 459-464. doi: $10.1080 / 01480545.2017 .1381110$

Li, I. C., Lee, L. Y., Tzeng, T. T., Chen, W. P., Chen, Y. P., Shiao, Y. J., et al. (2018b). Neurohealth properties of Hericium erinaceus mycelia enriched with Erinacines. Behav. Neurol. 2018:5802634. doi: 10.1155/2018/5802634

Li, I. C., Chen, Y. L., Chen, W. P., Lee, L. Y., Tsai, Y. T., Chen, C. C., et al. (2014a). Genotoxicity profile of erinacine A-enriched Hericium erinaceus mycelium. Toxicol. Rep. 1, 1195-1201. doi: 10.1016/j.toxrep.2014.11.009

Li, I. C., Chen, Y. L., Lee, L. Y., Chen, W. P., Tsai, Y. T., Chen, C. C., et al. (2014b). Evaluation of the toxicological safety of erinacine A-enriched Hericium erinaceus in a 28-day oral feeding study in Sprague-Dawley rats. Food Chem. Toxicol. 70, 61-67. doi: 10.1016/j.fct.2014.04.040

Li, I. C., Lee, L.-Y., Chen, Y.-J., Chou, M.-Y., Wang, M.-F., Chen, W.-P., et al. (2019). Erinacine A-enriched Hericium erinaceus mycelia promotes longevity in Drosophila melanogaster and aged mice. PLoS One 14:e0217226. doi: 10.1371/ journal.pone. 0217226

Mayo, C. D., Garcia-Barrera, M. A., Mazerolle, E. L., Ritchie, L. J., Fisk, J. D., Gawryluk, J. R., et al. (2019). Relationship between DTI metrics and cognitive function in Alzheimer's disease. Front. Aging Neurosci. 10:436. doi: 10.3389/ fnagi.2018.00436

Mcdonald, C. R., Ahmadi, M. E., Hagler, D. J., Tecoma, E. S., Iragui, V. J., Gharapetian, L., et al. (2008). Diffusion tensor imaging correlates of memory and language impairments in temporal lobe epilepsy. Neurology 71, 1869-1876. doi: 10.1212/01.wnl.0000327824.05348.3b

Mckhann, G., Drachman, D., Folstein, M., Katzman, R., Price, D., and Stadlan, E. M. (1984). Clinical diagnosis of Alzheimer's disease. Neurology 34:939.

Mori, K., Inatomi, S., Ouchi, K., Azumi, Y., and Tuchida, T. (2009). Improving effects of the mushroom yamabushitake (Hericium erinaceus) on mild cognitive impairment: a double-blind placebo-controlled clinical trial. Phytother. Res. 23 , 367-372. doi: 10.1002/ptr.2634

Mori, K., Obara, Y., Hirota, M., Azumi, Y., Kinugasa, S., Inatomi, S., et al. (2008). Nerve growth factor-inducing activity of Hericium erinaceus in 1321N1 human astrocytoma cells. Biol. Pharm. Bull. 31, 1727-1732. doi: 10.1248/bpb.31.1727

Nagano, M., Shimizu, K., Kondo, R., Hayashi, C., Sato, D., Kitagawa, K., et al. (2010). Reduction of depression and anxiety by 4 weeks Hericium erinaceus intake. Biomed. Res. 31, 231-237. doi: 10.2220/biomedres.31.231

Ng, T. K. S., Ho, C. S. H., Tam, W. W. S., Kua, E. H., and Ho, R. C.-M. (2019). Decreased serum brain-derived neurotrophic factor (BDNF) levels in patients with Alzheimer's Disease (AD): a systematic review and meta-analysis. Intern. J. Mol. Sci. 20:257. doi: 10.3390/ijms20020257

Ngandu, T., Lehtisalo, J., Solomon, A., Levalahti, E., Ahtiluoto, S., Antikainen, R., et al. (2015). A 2 year multidomain intervention of diet, exercise, cognitive training, and vascular risk monitoring versus control to prevent cognitive decline in at-risk elderly people (FINGER): a randomised controlled trial. Lancet 385, 2255-2263. doi: 10.1016/S0140-6736(15)60461-5

Nygard, L. (2003). Instrumental activities of daily living: a stepping-stone towards Alzheimer's disease diagnosis in subjects with mild cognitive impairment? Acta Neurol. Scand. Suppl. 179, 42-46.

Pan, X., Wu, X., Kaminga, A. C., Wen, S. W., and Liu, A. (2019). Dehydroepiandrosterone and dehydroepiandrosterone sulfate in Alzheimer's Disease: a systematic review and meta-analysis. Front. Aging Neurosci. 11:61. doi: 10.3389/fnagi.2019.00061 
Park, B.-Y., Lee, M. J., Kim, M., Kim, S.-H., and Park, H. (2018). Structural and functional brain connectivity changes between people with abdominal and nonabdominal obesity and their association with behaviors of eating disorders. Science 12:741. doi: 10.3389/fnins.2018.00741

Phan, C. W., David, P., and Sabaratnam, V. (2017). Edible and medicinal mushrooms: emerging brain food for the mitigation of neurodegenerative Diseases. J. Med. Food 20, 1-10. doi: 10.1089/jmf.2016.3740

Poleksic, A., and Xie, L. (2019). Database of adverse events associated with drugs and drug combinations. Sci. Rep. 9:20025. doi: 10.1038/s41598-019-56525-5

Poulose, S. M., Miller, M. G., Scott, T., and Shukitt-Hale, B. (2017). Nutritional factors affecting adult neurogenesis and cognitive function. Adv. Nutr. 8, 804-811. doi: 10.3945/an.117.016261

Saitsu, Y., Nishide, A., Kikushima, K., Shimizu, K., and Ohnuki, K. (2019). Improvement of cognitive functions by oral intake of Hericium erinaceus. Biomed. Res. 40, 125-131. doi: 10.2220/biomedres.40.125

Shimbo, M., Kawagishi, H., and Yokogoshi, H. (2005). Erinacine A increases catecholamine and nerve growth factor content in the central nervous system of rats. Nutr. Res. 25, 617-623.

Shohayeb, B., Diab, M., Ahmed, M., and Ng, D. C. H. (2018). Factors that influence adult neurogenesis as potential therapy. Transl. Neurodegen. 7:4. doi: 10.1186/ s40035-018-0109-9

Sindi, S., Kåreholt, I., Eskelinen, M., Hooshmand, B., Lehtisalo, J., Soininen, H., et al. (2018). Healthy dietary changes in midlife are associated with reduced dementia risk later in life. Nutrients 10:1649. doi: 10.3390/nu10111649

Spencer, S. J., Korosi, A., Layé, S., Shukitt-Hale, B., and Barrientos, R. M. (2017). Food for thought: how nutrition impacts cognition and emotion. Sci. Food 1:7. doi: 10.1038/s41538-017-0008-y

Sperling, R. A., Jack, C. R., and Aisen, P. S. (2011). Testing the right target and right drug at the right stage. Sci. Transl. Med. 3:111 cm133. doi: $10.1126 /$ scitranslmed. 3002609

Te Pas, M. F. W., Koopmans, S.-J., Kruijt, L., Calus, M. P. L., and Smits, M. A. (2013). Plasma proteome profiles associated with diet-induced metabolic syndrome and the early onset of metabolic syndrome in a pig model. PLoS One 8:e73087. doi: 10.1371/journal.pone.073087

Teare, M. D., Dimairo, M., Shephard, N., Hayman, A., Whitehead, A., and Walters, S. J. (2014). Sample size requirements to estimate key design parameters from external pilot randomised controlled trials: a simulation study. Trials 15:264. doi: 10.1186/1745-6215-15-264

Teng, E. L., Hasegawa, K., Homma, A., Imai, Y., Larson, E., Graves, A., et al. (1994). The cognitive Abilities screening instrument (CASI): a practical test for cross-cultural epidemiological studies of dementia. Int. Psychogeriatr. 6, 45-58. doi: $10.1017 / \mathrm{s} 1041610294001602$

Tzeng, T. T., Chen, C. C., Chen, C. C., Tsay, H. J., Lee, L. Y., Chen, W. P., et al. (2018). The cyanthin diterpenoid and sesterterpene constituents of Hericium erinaceus mycelium ameliorate alzheimer's disease-related pathologies in APP/PS1 transgenic mice. Int. J. Mol. Sci. 19:598. doi: 10.3390/ijms1902 0598

Conflict of Interest: Grape King Bio Inc., provided support in the form of salaries for the authors I-CL, W-PC, L-YL, Y-WC, Y-PC, and research materials, but did not have any additional role in the study design, data collection and analysis, decision to publish, or preparation of the manuscript.

The remaining authors declare that the research was conducted in the absence of any commercial or financial relationships that could be construed as a potential conflict of interest.

Copyright (C) 2020 Li, Chang, Lin, Chen, Lu, Lee, Chen, Chen, Chen and Lin. This is an open-access article distributed under the terms of the Creative Commons Attribution License (CC BY). The use, distribution or reproduction in other forums is permitted, provided the original author(s) and the copyright owner(s) are credited and that the original publication in this journal is cited, in accordance with accepted academic practice. No use, distribution or reproduction is permitted which does not comply with these terms. 